\title{
Uso del Análisis Factorial Exploratorio en RIDEP. Recomendaciones para Autores y Revisores
}

\author{
The Use of Exploratory Factor Analysis in RIDEP. Guidelines for Authors and \\ Reviewers
}

\author{
Rubén Daniel Ledesma ${ }^{1}$, Pere J. Ferrando ${ }^{2}$ y Jeremías David Tosi ${ }^{3}$
}

\begin{abstract}
Resumen
El Análisis Factorial Exploratorio (AFE) constituye una técnica de uso habitual en la investigación psicométrica. A pesar de su popularidad, las aplicaciones que hacemos los investigadores no siempre se ajustan a las recomendaciones de los expertos. En este trabajo se analizan 46 aplicaciones del AFE publicados en seis ejemplares de la RIDEP (42,2 a 47,2). Las aplicaciones se cotejan con las recomendaciones existentes en cuanto a: (a) la naturaleza de los datos a factorizar y la adecuación del AFE, (b) los procedimientos de extracción factorial, (c) los criterios para definir el número de factores, y (d) el tipo de método de rotación. Globalmente, se observa que las aplicaciones prácticas distan bastante de las recomendaciones. Se sugieren guías para autores y revisores. Esperamos que el trabajo contribuya al desarrollo de mejores prácticas en el uso del AFE.
\end{abstract}

Palabras clave: análisis factorial exploratorio, psicometría, revisión

\begin{abstract}
Exploratory Factor Analysis (EFA) is a widely used statistical technique in psychometrics research. Despite its popularity, real applications do not always fit the experts' recommendations. This paper analyses 46 applications of EFA published in RIDEP - volume/issue 42(2) to 47(2) -. These applications are compared with existing recommendations regarding: (a) the input data and the adequacy of the EFA, (b) the factor extraction procedures, (c) the number-of-factors-to-retain decision, and (d) the rotation methods. Overall, we observed that the real applications of EFA are far from the experts' recommendations. Guidelines for authors and reviewers are suggested. We hope this paper will promote best practices in EFA.
\end{abstract}

Keywords: exploratory factor analysis, psychometrics, review

\footnotetext{
${ }^{1}$ Lic. en Psicología y Dr. en Psicología. Profesor Titular/Investigador. IPSIBAT- Universidad Nacional de Mar del Plata y Consejo Nacional de Investigaciones Científicas y Técnicas, Buenos Aires, Argentina. Funes 3350, Mar del Plata (7600), Argentina. Tel.: +542234716015. Correo: rdledesma@conicet.gov.ar

${ }^{2}$ Lic. en Psicología y Dr. en Psicología. Catedrático/Investigador. CRAMC (Centro de Investigación para la Medida de la Conducta), Departamento de Psicología, Universitat Rovira i Virgili. Carretera Valls s/n. Tarragona (43007), España. Tel.:+34977558175. Correo: perejoan.ferrando@urv.cat

${ }^{3}$ Lic. en Psicología. Becario Doctoral. IPSIBAT- Universidad Nacional de Mar del Plata y Consejo Nacional de Investigaciones Científicas y Técnicas, Mar del Plata, Buenos Aires, Argentina. Funes 3350, Mar del Plata (7600), Argentina. Tel.: +542234716015. Correo: jeremiastosi@gmail.com
} 


\section{Introducción}

El AFE es una de las técnicas de análisis más difundidas y utilizadas con fines psicométricos. $\mathrm{Su}$ principal uso se orienta a explorar la dimensionalidad de las medidas y generar evidencias internas de validez, especialmente en las etapas iniciales de construcción o adaptación de instrumentos. Por esta razón, no es de extrañar que buena parte de los artículos publicados en RIDEP informen aplicaciones del AFE.

A pesar de ser una técnica muy popular en Psicología, algunos trabajos de revisión han indicado falencias y/o decisiones infundadas en las aplicaciones que los investigadores realizamos del AFE (e.g. Fabrigar, Wegener, MacCallum, \& Strahan, 1999; Frías-Navarro \& Pascual-Soler, 2012; Henson \& Roberts, 2006; Izquierdo \& Abad, 2014; Norris \& Lecavalier, 2010; Roberson, Elliott, Chang, \& Hill, 2014). Estos trabajos alertan sobre problemas asociados a la elección del procedimiento de extracción, los criterios para determinar el número de factores o la aplicación de métodos de rotación factorial, entre otros aspectos. Frente a esta situación, se han desarrollado numerosas guías $\mathrm{y}$ recomendaciones para propiciar mejores prácticas, algunas de las cuales se encuentran fácilmente accesibles al investigador iberoamericano en idioma castellano (e.g. Ferrando \& AnguianoCarrasco, 2010; Lloret-Segura, Ferreres-Traver, Hernández-Baeza, \& Tomás-Marco, 2014; Pérez \& Medrano, 2010) o portugués (Damásio, 2012). Sin embargo, los problemas parecen persistir, por lo que resulta conveniente reforzar las recomendaciones para autores y revisores.

Este artículo busca contribuir al desarrollo de mejores prácticas en la aplicación del AFE. Para ello, analizamos las aplicaciones publicadas en los últimos seis números de RIDEP y las cotejamos con algunas de las recomendaciones que han realizado los expertos. Los análisis no son exhaustivos, pero apuntan a cuestiones centrales. Estas cuestiones y algunas de las recomendaciones son descriptas de modo sintético en el próximo apartado. Sobre la base de los resultados obtenidos, sugerimos algunos lineamientos y guías para autores, revisores y editores de la RIDEP.

\section{Breve Revisión de Prácticas y Recomendaciones}

Un análisis completo de las decisiones que tomamos los investigadores al momento de aplicar un AFE y de las recomendaciones de los expertos excede los objetivos de este trabajo. Este análisis ha sido además bien desarrollado en trabajos previos (ver por ejemplo Lloret-Segura, FerreresTraver, Hernández-Baeza \& Tomás-Marco, 2014). No obstante, a continuación revisamos algunos puntos básicos que ayudarán a contextuar nuestro trabajo. En todo los casos, vale señalar que las recomendaciones no pueden considerarse "recetas" infalibles, sino más bien lineamientos generales para tomar decisiones más fundamentadas.

\section{Datos y Matrices de entrada}

La práctica tradicional y común consiste en analizar la matriz de inter-correlaciones Pearson entre items o elementos de un instrumento. Esta práctica parece propiciada por el hecho de ser la opción disponible en los programas más populares, como SPSS. No obstante, los expertos sugieren ser cautelosos con el uso indiscriminado de este enfoque, ya que puede ser problemático en ciertas circunstancias. En concreto, cuando los ítems tienen (a) distribuciones extremas (medias cercanas a los extremos de la escala de respuesta y distribuciones fuertemente asimétricas) y (b) alta capacidad discriminativa (véase e.g. Ferrando \& Lorenzo-Seva, 2014). En estas condiciones, el uso de correlaciones Pearson puede dar lugar a una inadecuada evaluación de la dimensionalidad (los llamados "factores de dificultad") así como a estimaciones distorsionadas de los pesos factoriales. El uso de pocas categorías de respuesta puede agravar aún más estos problemas ya que se añade entonces el problema de la atenuación por categorización. En la práctica estas circunstancias suelen ser más propias de ciertos dominios de investigación. Así, las medidas de personalidad o actitud no suelen presentar demasiados problemas, pero sí suelen hacerlo las de aptitud que contienen ítems muy fáciles y muy difíciles, y las clínicas o psicopatológicas que suelen contener ítems muy extremos y a la vez muy discriminativos.

La recomendación aquí sería evaluar si los datos cumplen con los criterios mínimos para 
justificar correlaciones Pearson (ítems no muy extremos y moderadamente discriminativos) o, en caso contrario (i.e. items politómicos con pocas opciones de respuesta y distribuciones sesgadas), considerar alternativas que se ajusten mejor a los datos, particularmente el análisis no lineal basado en correlaciones policóricas. Este último enfoque, sin embargo, tampoco está exento de dificultades. Según Ferrando (2014) las estimaciones basadas en correlaciones policóricas son menos precisas y estables, y en consecuencia pueden requerir muestras más grandes. En todo caso, la decisión sobre el tipo de matrices a factorizar parece requerir un análisis razonado de las distribuciones y características de los items en conjunción con otros factores (como el tamaño de la muestra). Este análisis debería ser informado como parte de los análisis previos a la aplicación de un AFE propiamente.

\section{Procedimiento de extracción}

Algunas de las críticas y recomendaciones más fuertes giran en torno al tipo de criterio o procedimiento elegido para estimar los factores. Entre las distintas opciones, Componentes Principales $(\mathrm{CP})$ sigue siendo en la práctica una de las más utilizadas (Frías-Navarro \& Pascual-Soler, 2012; Norris \& Lecavalier, 2010; Roberson, Elliott, Chang, \& Hill, 2014). Nuevamente, esto podría estar reforzado por el hecho de ser la opción por defecto en programas como SPSS. No obstante, hay mucho acuerdo en desaconsejar su uso, especialmente en contextos de análisis psicométrico. La razón es sencilla: $\mathrm{CP}$ no es un procedimiento para estimar un modelo factorial con error de medida, sino que es un método diseñado para obtener combinaciones lineales de las variables observables, sin diferenciar varianza común y varianza de error. Las consecuencias prácticas de ignorar el error de medida y utilizar $\mathrm{CP}$ como si fuese un procedimiento de extracción de factores son principalmente dos: (a) estimaciones sesgadas hacia arriba de los pesos factoriales y (b) sobreestimación de la dimensionalidad de la solución (e.g. Ferrando \& Anguiano-Carrasco, 2010). Si bien CP resulta computacionalmente menos demandante que otras opciones y además en ciertas circunstancias (muchos ítems con poco error de medida) podría arrojar una solución similar, hoy en día su uso no estaría justificado en la mayoría de los casos, máxime teniendo en cuenta que el error de medida de los ítems individuales es generalmente considerable. Los paquetes estadísticos ofrecen opciones más recomendables y el costo computacional ya no es un problema.

Dentro de los procedimientos que sí son estimadores del modelo factorial propiamente dicho existen opciones altamente recomendables, máxime cuando en las últimas décadas se han propuesto correcciones denominadas robustas que permiten una apropiada evaluación del ajuste (véase Ferrando \& Lorenzo-Seva, 2017). Para variables que pueden considerarse continuas (i.e. AF basado en la correlación de Pearson), el procedimiento de máxima verosimilitud robusto (MV-R) es una muy buena opción. Para variables tratadas como categóricas ordenadas (i.e. $\mathrm{AF}$ basado en policóricas), el procedimiento robusto de mínimos cuadrados ponderados es muy recomendable (WLS-R). Finalmente, tanto para variables continuas como categóricas, el procedimiento de mínimos cuadrados simples robusto (ULS-R), es, posiblemente, la mejor opción cuando las muestras no son grandes y el número de variables sí lo es.

\section{Determinación del Número de factores}

La determinación del número de factores es otro aspecto muy cuestionado en las aplicaciones del AFE (Norris \& Lecavalier, 2010, FríasNavarro \& Pascual-Soler, 2012). Si bien este es un tema más complejo e implica valoraciones a distinto nivel, también hay coincidencias en señalar problemas con ciertas prácticas. En particular, el uso de la regla de Kaiser o de los autovalores superiores a uno está totalmente desaconsejada. La principal razón es sencilla: el número de factores a retener según esta regla es proporcional al número de variables que se analizan y su uso tiende a sobreestimar el número de factores que son realmente relevantes e interpretables. A pesar de su inadecuación, sin embargo, la regla se sigue utilizando en la práctica.

A modo de síntesis, el enfoque más recomendable para una correcta determinación de la dimensionalidad de un conjunto de ítems, sería integral y múltiple. En primer lugar, utilizar medidas derivadas de la magnitud de los valores 
residuales tras extraer un número determinado de factores (raíz media cuadrática residual RMCR, distribución de los residuales, criterio de Kelley; véase e.g. Ferrando \& Anguiano-Carrasco, 2010). En segundo lugar, utilizar medidas o criterios auxiliares con una base y funcionamiento convincente, tales como el Análisis Paralelo, el MAP, el BIC o el método de Hull (Timmerman, Lorenzo-Seva, \& Ceulemans, 2018). Finalmente, utilizar criterios más sustantivos relacionados con la interpretabilidad y el grado de determinación de los factores resultantes (índice $\mathrm{H}$, o proporción de varianza común explicada; véase Ferrando \& Lorenzo-Seva, 2017).

\section{Métodos de Rotación}

Como en los casos anteriores, hay muchas opciones y métodos alternativos. La mayor distinción se realiza entre los métodos ortogonales y oblicuos, siendo los primeros los más comunes en la práctica (especialmente Varimax). No obstante, algunos expertos han cuestionado el uso generalizado y "por defecto" de esta opción, pues debería estar supeditada al análisis de la relación entre los factores (Ferrando, 2010). En efecto, los métodos ortogonales, habituales en la práctica, tendrían sentido cuando los factores se suponen no relacionados en la teoría. Sin embargo, lo habitual es que las subdimensiones que integran los constructos psicológicos se encuentren relacionadas en mayor o menor medida. En este sentido, los métodos oblicuos serían generalmente más adecuados y deberían ser considerados como primera opción en el caso puramente exploratorio (Browne, 2001). Si la solución obtenida indica correlaciones negligibles entre factores siempre puede pasarse a una solución ortogonal (más parsimoniosa) en una segunda fase.

\section{Método}

Se realizó un análisis inicial del contenido de todos los artículos publicados en los seis números finales de RIDEP al momento de elaborar este trabajo (Junio de 2018). Sobre esa base, se identificaron 36 artículos que informaban al menos un AFE. Dentro de un mismo artículo, cada AFE fue analizado y contabilizado por separado (excepto en un artículo donde se informan 8 AFE, uno para cada sub-escala del mismo instrumento, y se sigue en todos los casos el mismo procedimiento). De ese modo, se registraron $\mathrm{y}$ analizaron un total de 46 aplicaciones del AFE. Para cada AFE, se evaluaron diferentes aspectos. Primero, se registraron algunas cuestiones básicas del diseño (como el tamaño de la muestra, la cantidad de variables, el uso independiente o combinado con un Análisis Factorial Confirmatorio - AFC -, y el software estadístico utilizado). Segundo, se analizó el tipo de datos de entrada y el tipo de matriz de correlaciones a factorizar, así como la existencia de comprobaciones previas sobre las distribuciones de las variables y la adecuación del AFE (e.g. aplicación y valores del test de Kaiser, Meyer, Olkin - KMO). Tercero, se registró el tipo de método de extracción y rotación utilizada (ortogonales $\mathrm{u}$ oblicuas en sus diferentes versiones), y los criterios aplicados para definir el número de factores a retener (autovalores superiores a uno, Scree Plot, Análisis Paralelo, etc.). Complementariamente, se observó el modo en que se informan las tablas de resultados con pesos factoriales. Por último, los resultados de las aplicaciones del AFE son comparados con los lineamientos sugeridos en trabajos previos, especialmente Ferrando y Anguiano-Carrasco (2010), Ferrando y Lorenzo-Seva (2014) y Lloret et al. (2014).

\section{Resultados}

En la mayoría de los casos el AFE fue aplicado a nivel de items (95\%) y en menor medida a nivel de escalas. Dentro del primer grupo, un $31 \%$ corresponden a AFEs aplicados independientemente a distintas sub-escalas o partes de un mismo instrumento. El $89.2 \%$ de los análisis se aplicaron a muestras de tamaño mayor a 200 casos $(n<200=11.1 \%, n$ entre $201-300=$ $26.7 \%, n$ entre $301-600=40 \%, n>601=22.2 \%)$. Un $36.9 \%$ de los AFEs se aplican como paso previo a un AFC. La mitad de las aplicaciones se realizan sobre datos cuya razón $n$ /variables es menor a 10 (mínimo: 2.88; Mediana: 10.22; Máximo: 142). El software más utilizado es SPSS, como programa principal o en combinación con otros $(58.7 \%)$. FACTOR es mencionado en el $17 \%$ de los casos. 
Un $24 \%$ no informan el software utilizado. Entre los programas referenciados también se cuentan Mplus, Stata y R.

Más del $90 \%$ de los artículos analizan items de respuesta graduada, siendo el formato más común el modelo Likert de 5 puntos. En cuanto al tipo de matrices que se factorizan, en el $74 \%$ de los análisis no se informa (aunque es de suponer que se trata de matrices Pearson). Un 22\% mencionan el uso de matrices policóricas. Solo un $13.3 \%$ de los trabajos analizan e informan aspectos importantes de las distribuciones de los items, como índices de asimetría y curtosis. Un 9\% mencionan estos análisis, pero no muestran los resultados. La gran mayoría (78\%) no ofrece ni menciona estos análisis como tarea previa al AFE. Por otro lado, gran parte de los trabajos informan resultados para el Test de Bartlett y el test KMO (un $24 \%$ no brindan esta información). Entre los que informan $\mathrm{KMO}$, la mayoría tiene valores superiores a $.80(74 \%)$.

En relación a los métodos de extracción de factores, más de la mitad utilizan Componentes Principales (58\%). Otros métodos informados en menor medida fueron: Ejes Principales, Máxima Verosimilitud y Mínimos Cuadrados No Ponderados - ULS. En muy pocos casos se justifica explícitamente la elección del método de extracción. Un $11 \%$ no ofrecen información sobre este aspecto del análisis. En cuanto a los criterios para definir el número de factores, el Scree test es mencionado como al menos uno de los métodos utilizados en el $36 \%$ de las aplicaciones. No obstante, en pocos casos se muestra el gráfico $\mathrm{y}$, cuando se presenta, se observan decisiones objetables e incluso visiblemente erróneas. Por otro lado, la regla de los autovalores superiores a uno es mencionada en un $20 \%$ de las aplicaciones como uno de los criterios utilizados. Muy pocos trabajos aplican métodos más objetivos, siendo en este caso el Análisis Paralelo el más utilizado (17\%). El uso comprensivo de varios criterios (estadísticos, de la solución y teóricos) se menciona en pocas ocasiones. En un $20 \%$ de las aplicaciones no se informa nada sobre cómo se determinó el número de factores.

En lo referente a los métodos de rotación, el $48 \%$ corresponde a métodos ortogonales (principalmente Varimax), el $43 \%$ a métodos oblicuos (principalmente PROMAX), un $4 \%$ no informan el tipo de rotación, y el resto son soluciones unidimensionales. Cuando se usan rotaciones ortogonales, no se observan justificaciones explícitas de esta decisión frente a las alternativas oblicuas, y en la mayoría de los casos no se informan las correlaciones entre factores. En algunos casos, se esperan teóricamente factores relacionados o se informan altas correlaciones entre factores, pero esta situación no parece influir sobre la decisión acerca del tipo de rotación seleccionada.

Por último, pocos trabajos ofrecen información completa sobre lo pesos factoriales de las variables en todo los factores (29\%). Un $44 \%$ de las aplicaciones muestran matrices con pesos que superan algún valor $\left(\begin{array}{lll}.30 & 0 & .40\end{array}\right.$ usualmente), y un $27 \%$ directamente no ofrecen información alguna.

\section{Discusión}

Los resultados sugieren la necesidad de promover mejores prácticas en la aplicación del AFE. En primer lugar, se observan omisiones importantes en la presentación de resultados, lo que limita las posibilidades de evaluación y réplica. Por ejemplo, un tercio de los análisis no informan al menos uno de los siguientes aspectos: método de extracción, método de rotación o criterios para la selección de factores. Por otro lado, las decisiones sobre el modo en que se aplican los AFEs son en muchos casos cuestionables o infundadas, y conducen a procedimientos desaconsejados por los expertos. A continuación, sintetizamos algunos de los puntos que a nuestro juicio merecen ser atendidos $\mathrm{y}$ ofrecen algunas sugerencias para autores, revisores y editores.

Un punto débil de las aplicaciones del AFE en RIDEP es la falta de información suficiente a nivel de los items/variables, en las fases previas de comprobación (e.g. medidas de posición y forma, número de categorías, etc.), pero también en los informes de resultados (e.g. pesos factoriales y comunalidades). En un primer momento, esta información es relevante para juzgar adecuadamente las decisiones y alternativas, especialmente el tipo de matriz a factorizar. Vale mencionar que solo una pequeña 
fracción de trabajos ofrecía esta información y justificaban el uso de una determinada matriz de correlación (matrices policóricas en todos los casos). Por otro lado, al momento de presentar los resultados también se observaron importantes omisiones. Por ejemplo, menos de un tercio de los casos ofrecían matrices mostrando los pesos factoriales en todos los factores, e incluso una importante fracción no presentaba ninguna matriz factorial. Como ha sido sugerido en trabajos previos (Frías-Navarro \& Pascual-Soler, 2012), la información completa sobre los pesos factoriales y comunalidades es clave para evaluar el significado y calidad de la solución.

Otras cuestiones relevantes se refieren a las decisiones sobre los métodos de extracción y rotación factorial, y a los procedimientos para determinar un número de factores. Por ejemplo, Componentes Principales es la opción más utilizada entre los trabajos analizados, aunque su uso ha sido desaconsejado para fines psicométricos. Las rotaciones ortogonales, en especial Varimax, también se siguen aplicando de un modo algo injustificado, incluso en casos donde los resultados indican que una rotación oblicua hubiese sido probablemente una mejor opción. En la determinación del número de factores también se observan prácticas poco recomendadas (uso de la regla de los autovalores superiores a uno), poco transparentes (decisiones basadas en gráficos que no se presentan) e incluso decisiones visiblemente erróneas. En general, parecen primar las opciones por defecto del software más popular (i.e., SPSS), a pesar de no ser las más convenientes. Como ha sido señalado en otros trabajos (ver e.g. Lloret, Ferreres, Hernández, \& Tomás, 2017), la influencia del software estadístico en la aplicación del AFE parece clave, y debería propiciarse un uso más reflexivo e informado en este sentido.

Vale mencionar que las observaciones previas sobre las prácticas del AFE en RIDEP están en línea con otros estudios que han señalado problemas similares en distintas áreas de aplicación de la Psicología (Fabrigar, Wegener, MacCallum, \& Strahan, 1999; Frías-Navarro \& Pascual-Soler, 2012; Henson \& Roberts, 2006; Izquierdo \& Abad, 2014; Norris \& Lecavalier, 2010; Roberson, Elliott, Chang, \& Hill, 2014). Entendemos que será necesario un esfuerzo conjunto de autores, revisores y editores para propiciar mejores prácticas en el uso de este método. Los autores de RIDEP debemos actualizar nuestros conocimientos para lograr mejores aplicaciones. Guías y recomendaciones muy asequibles al investigador en Psicología se encuentran publicadas en trabajos previos (e.g. Damásio, 2012; Ferrando \& Anguiano-Carrasco, 2010; Lloret-Segura, Ferreres-Traver, HernándezBaeza \& Tomás-Marco, 2014; Pérez \& Medrano, 2010). Por su lado, los revisores de las contribuciones deberían observar de modo más crítico las aplicaciones del AFE, solicitando mayor información sobre las decisiones y resultados. Eventualmente, sería importante que sugieran a los editores una evaluación estadística complementaria si no se dispone de la experticia suficiente.

Será clave además que los editores incentiven el cumplimiento de recomendaciones básicas en la aplicación del AFE. Es importante que ofrezcan guías prácticas y se aseguren las condiciones necesarias para lograr mejores y más completos informes de resultados (e.g. facilitar la inclusión de anexos estadísticos si es conveniente). Creemos además que sería muy positivo propiciar una política editorial tendiente a la publicación de los datos originales, de manera que los análisis pudieran ser replicados. Por último, incluimos como Anexo a este artículo una guía básica sobre AFE, orientada principalmente a autores $y$ revisores de RIDEP. Esperamos que esta guía ayude a promover mejores prácticas en la aplicación y evaluación del AFE.

\section{Referencias}

Damásio, B. F. (2012). Uso da análise fatorial exploratória em psicologia. Avaliação Psicológica, 11(2) 213-228

Fabrigar, L. R., Wegener, D. T., MacCallum, R. C., \& Strahan, E. J. (1999). Evaluating the use of exploratory factor analysis in psychological research. Psychological Methods, 4, 272-299.

Ferrando, P. J., \& Lorenzo-Seva, U. (2017). Program FACTOR at 10: Origins, development and future directions. Psicothema, 29(2), 236-240. 
Ferrando, P. J., \& Anguiano-Carrasco, C. (2010). El análisis factorial como técnica de investigación en psicología. Papeles del Psicólogo, 31(1), 18-33

Ferrando, P. J., \& Lorenzo-Seva, U. (2014). El análisis factorial exploratorio de los ítems: Algunas consideraciones adicionales. Anales de Psicología, 30(3), 1170-1175.

Frías-Navarro, D., \& Pascual-Soler, M. (2012). Prácticas del Análisis Factorial Exploratorio (AFE) en la investigación sobre conducta del consumidor y marketing. Suma Psicológica, 19(1), 45-58.

Henson, R. K., \& Roberts, K. (2006). Use of exploratory factor analysis in published research common errors and some comment on improved practice. Educational and Psychological Measurement, 66(3), 393-416

Izquierdo, I., Olea, J., \& Abad, F. J. (2014). El análisis factorial exploratorio en estudios de validación: Usos y recomendaciones. Psicothema, 26(3), 395-400.

Lloret-Segura, S., Ferreres-Traver, A., \& TomásMarco, I. (2017). El análisis factorial exploratorio de los ítems: Análisis guiado según los datos empíricos y el software. Anales de Psicología, 33(2), 417-432.

Lloret-Segura, S, Ferreres-Traver, A., HernándezBaeza, A., \& Tomás-Marco, I. (2014). El análisis factorial exploratorio de los ítems: Una guía práctica, revisada y actualizada. Anales de Psicología, 30, 3, 1151-1169

Norris, M., \& Lecavalier, L. (2010). Evaluating the use of exploratory factor analysis in developmental disability psychological research. Journal of Autism and Developmental Disorders, 40, 1, 8-20.

Pérez, E. R., \& Medrano, L. (2010). Análisis factorial exploratorio: Bases conceptuales y metodológicas. Revista Argentina de Ciencias del Comportamiento, 2(1) 58-66

Roberson, R. B. III, Elliott, T. R., Chang, J. E., \& Hill, J. N. (2014). Exploratory factor analysis in rehabilitation psychology: A content analysis, Rehabilitation Psychology, 59(4), 429-438. 
Anexo 1 - Análisis Factorial Exploratorio - Guía de Evaluación.

Esta guía ofrece algunas pautas generales para informar y evaluar aplicaciones del Análisis Factorial Exploratorio.

\section{Preguntas Básicas}

1. Datos básicos de ¿Se justifica el objetivo y aplicación del AFE?, ¿Se informan claramente la naturaleza la aplicación de las variables y/o el formato de respuestas de los items?, ¿Se informa el tamaño y características de la muestra sobre la que se ejecuta el AFE?.

Nota: Todos estos puntos deberían ser fácilmente identificables.

2. Datos de entrada y Análisis Preliminares
¿Se informan estadísticos descriptivos a nivel de las variables/items (medidas de posición, variabilidad y forma)?, ¿Se informa y justifica el tipo de matriz de correlación que se factoriza (correlaciones Pearson, Policóricas, Tetracóricas)?, ¿Se presentan índices de adecuación (e.g. KMO) con valores aceptables?.

Nota: Se desaconseja el uso por defecto de correlaciones Pearson, sin una previa exploración de las distribuciones y características de los items. En caso de correlaciones policóricas, se recomiendan tamaños muestrales de al menos n=200.

\section{Métodos de Extracción}

¿Qué método de extracción factorial se utiliza?, ¿Se justifica su elección?.

Nota: Se desaconseja el uso de Componentes Principales como método de extracción factorial. Se sugieren MV-R (Pearson) y WLS-R (policóricas). Para ambos casos, cuando la muestra es moderada y el número de variables grande, se sugiere ULS-R.

\section{Número de factores}

¿Se mencionan los criterios utilizados para determinar el número de factores a retener?, ¿La elección final del número de factores está bien justificada y se apoya en criterios múltiples?.

Nota: Se desaconseja el uso automático de la regla de Kaiser (autovalores superiores a uno). Se sugiere el uso de métodos alternativos (MAP, RMCR, Análisis Paralelo, Índices de ajuste), en conjunción con otros aspectos de la solución (número mínimo de ítems por factor, configuración e interpretabilidad teórica de la solución, etc.). En caso de usar un Scree Plot, se debería incluir el gráfico.

\section{Métodos de Rotación}

En caso de soluciones de dimensionalidad mayor a uno (más de un factor): ¿Se aplica un método de rotación?, ¿Se informa y justifica el tipo de rotación?, ¿Se incluyen en los resultados las correlaciones entre factores?.

Nota: Se desaconseja usar rotaciones ortogonales (e.g. Varimax) como método por defecto. Si se esperan/observan factores correlacionados se aconseja el uso de rotaciones oblicuas (especialmente en correlaciones entre factores superiores a .30).

\section{Tablas con Matrices de pesos factoriales}

\section{Se sugiere que la tabla incluya:}

(1) Los pesos de todos los items en todos los factores, remarcando en negrita los valores superiores a un criterio (e.g. .30 o .40),

(2) Una columna final con las comunalidades de los items,

(3) En la parte inferior (luego de los pesos): la varianza explicada por cada factor y la varianza total, los valores de KMO y la prueba de Bartlett.

(4) Una nota a pie de Tabla indicando: método de extracción, tipo de rotación y tipo de matriz factorizada. 